\title{
Leserkommentar zur
}

\section{«Praxis Nr. 3»: Die Ökonomisierung der Medizin}

Mit Interesse habe ich die «Praxis»-Ausgabe zur «Ökonomisierung der Medizin» durchgelesen und freue mich über das Interesse der Redaktion an Leserkommentaren.

Im Grossen und Ganzen war ich von den Beiträgen eher enttäuscht, aber nicht überrascht. An idealistischen Bekundungen und technokratischen System-Verbesserungsideen (PROMs, TARCIO etc.) wurde nicht gespart. Einzig Herr Schwarz hat versucht, das zugrundliegende Missverständnis gegenüber Märkten zu klären und auf Probleme etatistischer Lösungen hinzuweisen. Da das Outcome auf Märkten unvorhersehbar ist, verzichtete er auf idealistische oder utopische Ideen. Verwunderlich ist das Ganze nicht, denn der Begriff Ökonomisierung ist ziemlich schwammig, ebenso wie «Gesundheitswesen», «Medizin», «Gesundheit» und «Krankheit». Keiner der Autorinnen und Autoren hat auf die beiden Grundformen des Wirtschaftens, nämlich das bürokratische Wirtschaften der Verwaltung und das betriebswirtschaftliche Wirtschaften privater Unternehmen, unterschieden. Beide Formen haben ihren Sinn und Zweck, je nach Umfeld fällt Ökonomisieren anders aus. Das Ökonomisieren privater Akteure (z.B. Ärzte) auf Kosten eines Drittfinanzierers zu kontrollierten Preisen und unter dem Schutz eines Lizenzmonopols ist natürlich ein bizarre Zwischenform, welche verständlich zu viel Unmut und ebenso bizarren Resultaten führt.

Konkret hätte von Produktivitätssteigerung, Effizienzsteigerung, Kommodifizierung oder Verbetriebswirtschaftlichung je nach Umfeld - Markt oder Verwaltung gesprochen werden müssen. Ebenso wenig wurden die verschiedenen ökonomischen Denkschulen unterschieden. Es gibt eben nicht die eine richtige Ökonomie als Wissenschaft, sondern - wie in der Medizin - verschiedene Richtungen. Klassisch Liberale Ökonomen verstehen die Wirtschaft als Sozialphilosophie und orientieren sich an der subjektiven Wertlehre, während sich neoklassische Ökonomen à la Keynes auf Zahlen fixieren und diese anhand mathematischer Modelle (Kosten-Nutzen-Rechnungen) zugunsten staatlicher Markteingriffe produzieren. Die Unterschiede könnten nicht grösser sein, Marktwirtschaft oder Planwirtschaft (bzw. «Versorgungssystem»)? Was die Letzteren und heutigen MainstreamÖkonomen angerichtet haben, lässt sich gut anhand der Misere der Zentralbanken veranschaulichen. $\mathrm{Zu}$ viele Ökonomen verderben wohl den Brei. Interessanterweise war ja die Sovjetunion das Land mit der grössten Anzahl Ökonomen.
Aber genug von meiner Meinung, denn in der wirtschaftlichen Realität der Märkte zählen nicht Meinungen, sondern Handlungen. Diese spiegeln sich auf dem Markt in Form von Angeboten und Preisen wider. Preise sprechen eine eigene Sprache, sie setzen Anreize und signalisieren Informationen. Sie sind Ausdruck realer Entscheidungen und besitzen eine Koordinationsfunktion. Ein bezahlter Preis ist schon mal ein Feedback, im Gegensatz $\mathrm{zu}$ einer momentanen Kundenmeinung. Erst auf dem Markt können Einzelne einen realen Nutzen für Andere (Fremde) anbieten und davon leben. Nicht besser meinen, sondern besser tun. Genial, nicht? Anders im Gesundheitswesen. Die ständigen Systemdiskussionen sind eben daher völlig nutzlos, da sich Systemveränderungen in diesem hochregulierten Sektor nur politisch bewerkstelligen lassen. Somit wird die Politik und das damit verbundene Lobbying der Interessengruppen zum einzig realen Instrument der Veränderung, was nichts anderes als tragisch ist. Praktisch gesehen gibt es nur zwei Richtungen aus der Sackgasse: Liberalisierung/Privatisierung oder Zentralisierung/Verstaatlichung.

Um für den liberalen Weg und damit auch die Autonomie der Ärzte zu sprechen, knüpfe ich an Herrn Schwarz an und weise auf zwei konkrete Beispiele hin, die zeigen, dass «Ökonomisieren» auf dem freien Markt auch für Ärzte funktionieren kann, und zwar ohne Drittfinanzierer und Versicherungszwang:

Direct Primary Care: Eine Bewegung von Grundversorgern in den USA, die unabhängig von Krankenkassen arbeiten. Die Finanzierung funktioniert über Mitgliederbeiträge der Patienten an den Arzt/ein Ärztenetzwerk. Die Ärzte nehmen die Preisgestaltung selbst in die Hand, und siehe da, es funktioniert! www.dpcare.org

Das Surgery Center Oklahoma gilt als Beispiel dafür, dass auch teuere chirurgische Eingriffe zu kompetitiven Marktpreisen möglich sind, unabhängig von staatlicher Subventionierung und Krankenkassen. Die Zufriedenheit der Patienten ist hoch und die Preise im Vgl. zu den Kassen-Kliniken oft um ein Vielfaches tiefer: https://surgery centerok.com.

Das Liberale Institut mit Sitz in Zürich hat zwei Bücher mit weiteren konkreten Vorschlägen aus dem politisierten Gesundheitskollektivismus (z.B. Gesundheitssparkonten, Hochrisikoversicherung) herausgegeben. Wer sich für konkrete Idee interessiert ist, die nicht auf reiner Umverteilung basieren, wird dort fündig: https://www.libinst.ch/?i=zuteures-gesundheitswesen. 
Ich wünsche mir gerade für die junge Ärztegeneration einen differenzierteren und offeneren Zugang zum Thema Ökonomie und Medizin, damit sie nicht gänzlich zum Spielball der Politiker und Interessengruppen (Versicherer, Ärzteverbände, Gesundheitsökonomen etc.) werden.
Dr. med. Marc Fouradoulas

FMH Allgemeine Innere Medizin Psychosomatik, SAPPM

MAS Managed Healthcare

Badenerstrasse 75

8004 Zürich

mfouradoulas@hin.ch 